\title{
Personalia
}

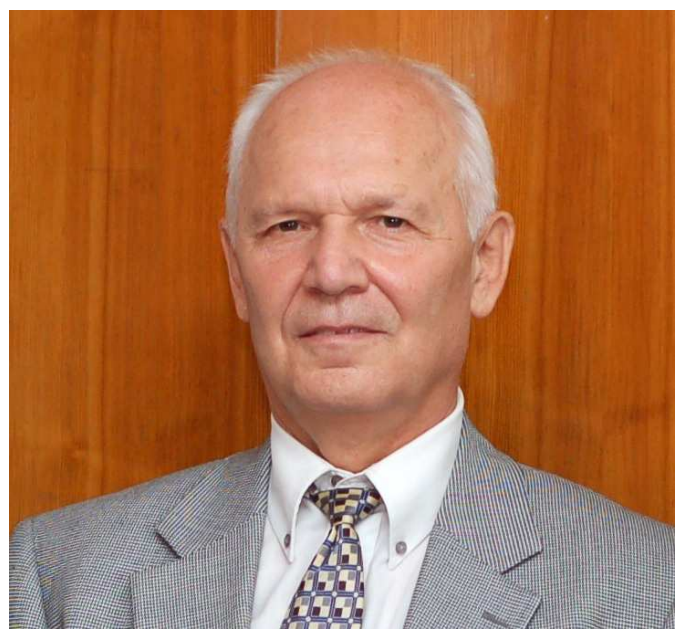

\section{Vadim Loktev's 70th birthday}

This year Vadim Loktev, a well-known scientist, prominent theoretical physicist, talented lecturer, organizer of scientific research and activity celebrates his 70th birthday. Vadim Loktev was born on May, 3, 1945 in Kyiv (Ukraine); his parents were professional employees. He studied at the Department of Physics of Taras Shevchenko State University (Kyiv). After graduation from the university in 1968 he was a postgraduate student at the Institute for Theoretical Physics of the Academy of Sciences of the Ukrainian SSR [now Bogolyubov Institute for Theoretical Physics of the National Academy of Sciences of Ukraine (NASU)] in 1968-1971. He has been working at this institute since 1967 up to the present time now occupying the position of Head of the Department of Nonlinear Condensed Matter Physics. In 1971 V. Loktev defended his Ph.D. thesis under the supervision of the world-renowned scientist A. Davydov; in 1983 he defended his D.Sc. thesis. In 1997 he obtained title of Professor. The same year V. Loktev was elected a corresponding member and in 2003 he was elected a full member of the NASU.

Solid state physics, especially the theory of optical effects, the physics of magnetic phenomena, the processes in disordered media, and the problems of high-temperature superconductivity are the main fields of V. Loktev's scientific interests. The scientific style of V. Loktev, a bright representative of Davydov's school, is clearly manifested in these areas. His focus in Physics is to elucidate and describe the physical mechanism of investigated phenomena in conjunction with adequate theoretical tools, to solve the actual problems which are on the first line of scientific search. Among Loktev's early scientific achievements we can emphasize the investigation of biexitonic splitting and polarization of spectral lines in the region of two-particle absorption, which enabled one to explain the features of the $\alpha$-oxygen optical spectra (with A. Prikhot'ko and Yu. Gaididei). The magnetic phases of solid oxygen were studied and, in particular, the magnetic structure of the low-temperature $\beta$-phase was predicted (the so-called "Loktev's structure”). Developing the quantum theory of phenomena in magnetic crystals with a strong magnetic anisotropy, V. Loktev (with V. Ostrovskii) predicted and described a new magneto-optical effect, whose essence consists in a linear dependence of dielectric susceptibility components on the magnetic field (for the systems where the anisotropy energy is of the same order as the value of exchange interaction). Together with M. Ivanov and Yu. Pogorelov, V. Loktev developed a general approach in the theory of disordered magnetics aimed at the description of long-range impurity states.

V. Loktev has brought a substantial contribution to the theory of superconducting systems. The growing attention to this area was stimulated in the last decades of the 20th century by the discovery of hightemperature superconductivity (HTSC). V. Loktev actively participated in these investigations. In collab- 
oration with $\mathrm{Yu}$. Gaididei, his attention was paid to the new nonphononic mechanism of electron pairing in HTSC. The Bardeen-Cooper-Schrieffer theory was extended by V. Loktev (with V. Gusynin and S. Sharapov) to metals with the variable carrier concentration. It enabled one to describe a crossover between the Bose-Einstein superfluidity of electron pairs and the superconductivity as such and to clarify in this framework the reason of appearing a pseudogap in the spectrum. The Jahn-Teller mechanism of electron pairing in fullerites was investigated as well (together with E. Pashitskii).

Being interested in the physics of antiferromagnets, V. Loktev has recently performed a set of investigations (with H. Gomonai) devoted to the theory of the domain creation in antiferromagnetic materials and to the description of effects occurring in such systems at the presence of spin-polarized current. The effect of the torque moment appearance was predicted in such a case.

The scientific activity of V. Loktev, as a member of authors research teams, was distinguished by two State Prizes of Ukraine in Science and Technology (1977 and 1990) for the investigation of elementary excitations in cryocrystals and the study of nonideal magnetics with paramagnetic impurities. He was a recipient of two annual awards in science of the NASU (Sinelnikov Prize, 1985, and Bogolyubov Prize, 2006), possesses the honorary title "Honored Worker in Science and Technology of Ukraine" (2000). The European Academy of Sciences, Art and Literature elected V. Loktev its full member. In 2015 he was awarded (together with A. Abrikosov) the Vernadsky Gold Medal of the NASU for outstanding achievements in the field of high-temperature superconductivity. The activity of V. Loktev has been distinguished by state rewards.

V. Loktev devotes considerable attention to the education and training of students and young scientists. For 15 years (since 1982) he was a lecturer at the Faculty of Physics, Electronics and Computer Systems of Taras Shevchenko National University of Kyiv; since 1998 he has been Head of the Department of General and Theoretical Physics at the National Technical University "Kyiv Polytechnic Institute”. Under his supervisory the $11 \mathrm{Ph} . \mathrm{D}$. theses and 4 D.Sc. theses have been defended. V. Loktev is the author of over 300 scientific publications, among them 3 monographs and 3 textbooks.

An important and considerable part of V. Loktev's work is the organizing activity in the field of scientific research. He is a member of the International advisory council of the "Low Temperature Physics" journal, a member of several scientific editorial boards. He actively works in research councils of the National Academy of Sciences of Ukraine and of the Ministry of Education and Science of Ukraine. Starting from 2004 V. Loktev has been the academician-secretary of the Physics and Astronomy Division of the NASU. Since 2013 he has been the editor-in-chief of the Great Ukrainian Encyclopedia.

In addition, it should be mentioned that V. Loktev is a well-known publicist and promoter of scientific knowledge. He cares for the perspectives of further development of science and education in Ukraine, for the current problems and events taking place in our country. In this connection, he expresses his opinions and reflections in periodicals and other mass media.

Vadim Loktev is an active and determined person able to solve various complex problems. Serving as an example to follow and promoting innovatory ideas he inspires people around him and his colleagues. People communicating with V. Loktev always feel his positive influence.

On the occasion of the 70th birthday, the Editorial Board of the "Condensed Matter Physics" journal congratulates Professor V. Loktev and wishes him good health, further successes in science and life and new creative achievements. 